\title{
Various Scattering Properties of a New PT-symmetric non-Hermitian potential
}

\author{
Ananya Ghatak* Raka Dona Ray Mandal ${ }^{\dagger}$ and Bhabani Prasad Mandal $\ddagger$ \\ Department of Physics, Banaras Hindu University, Varanasi-221005, INDIA. \\ $\dagger$ Department of Physics, Rajghat Besant School, Varanasi-221001, INDIA.
}

\begin{abstract}
We complexify a 1-d potential $V(x)=V_{0} \cosh ^{2} \mu\{\tanh [(x-\mu d) / d]+\tanh (\mu)\}^{2}$ which exhibits bound, reflecting and free states to study various properties of a non-Hermitian system. This potential turns out a PT-symmetric non-Hermitian potential when one of the parameters $(\mu, d)$ becomes imaginary. For the case of $\mu \rightarrow i \mu$, we have entire real bound state spectrum. Explicit scattering states are constructed to show reciprocity at certain discrete values of energy even though the potential is not parity symmetric. Coexistence of deep energy minima of transmissivity with the multiple spectral singularities (MSS) is observed. We further show that this potential becomes invisible from left (or right) at certain discrete energies. The penetrating states in the other case $(d \rightarrow i d)$ are always reciprocal even though it is PT-invariant and no spectral singularity (SS) is present in this case. Presence of MSS and reflectionlessness are also discussed for the free states in the later case.
\end{abstract}

*e-mail address: gananya04@gmail.com

${ }^{\dagger}$ e-mail address: rakad.ray@gmail.com

‡e-mail address: bhabani.mandal@gmail.com, bhabani@bhu.ac.in 


\section{Introduction}

PT-symmetric non-Hermitian system [1] with real energies are very exciting, because one can have fully consistent quantum theory by restoring the Hermiticity and by upholding the unitary time evolution for such systems in a modified Hilbert space [2,3]. These interesting results help this subject to grow exponentially and to find many applications in different branches of physics [4]-[22]. Experimental realizations of such systems in optics have further enhanced the motivations for such studies [20, 21, 22]. However not all PT-symmetric non-Hermitian systems lead to fully consistent quantum theory because of the presence of exceptional points (EP) [23, 24, 25] and spectral singularities (SS) $[26,27,29]$ where two or more eigenvalues along with corresponding eigen functions coalesce. This phenomenon is known to have physically observable consequences. Scattering cross-section and/ or reflection and transmission current diverges. Thus it is extremely important to study the presence of such singular points in non-Hermitian systems.

For any real or complex potential, reflectivity $(R)$ and transmissivity $(T)$ do not depend on the direction of incidence as long as the potential remains invariant under parity transformation. PT-symmetric non-Hermitian potentials are naturally parity non-invariant and hence the reflection coefficients for left $\left(R_{l}\right)$ incident particle and right $\left(R_{r}\right)$ incident particle are generally not equal to each other, i.e. $R_{l} \neq R_{r}$, even though $T_{l}=T_{r}=T$. Thus all the PT-symmetric non-Hermitian potentials are non-reciprocal. In other word they show handedness [29]. However at certain discrete energies these PT-symmetric non-Hermitian potentials become reciprocal, i.e. $R_{l}=R_{r}$ and/or they become unitary $(R+T=1)$. It is extremely important to find such energy points to get further insight into these systems.

Invisibility of a complex potential is another important aspect of scattering [16, 30, 31]. The potential is called invisible from left if $R_{l}=0, R_{r} \neq 0$ and in addition $T=1$; similarly the potential become invisible from right if $R_{r}=0, R_{l} \neq 0$ and $T=1$. Recently it has been shown that the equations governing invisibility of the potential are invariant under combined parity and time reversal transformation [30]. However PT-symmetry is neither necessary nor sufficient for the invisibility of a scattering potential. The concept of invisibility could lead to many applications in optics where analogy is made between complex potential and complex refractive index of a optical material. The efficiency of optical device is enhanced by reducing the level of absorption [32, 33].

The purpose of the present work is to study various aspect of these scattering properties $[34,35]$ by considering a new PT-symmetric non-Hermitian potential. We consider a 1-d real potential [36]

$$
V(x)=V_{0} \cosh ^{2} \mu\{\tanh [(x-\mu d) / d]+\tanh (\mu)\}^{2}
$$

which admits bound, reflecting and free state solutions depending on the energy of the particle. $V_{0}$ is the depth of the potential, the parameter d controls the width of the potential and $\mu$ decides whether the potential behaves like a well or a barrier. We complexify this potential in PT-symmetric manner by allowing the parameters $(\mu, d)$ to become imaginary separately. We show that entire bound state spectrum is real and the system always 
remains in unbroken phase of PT-symmetry when $\mu \rightarrow i \mu$. By considering explicit scattering states we show that reciprocity is restored even for a PT-symmetric non-Hermitian system at certain discrete values of energy. This system has multiple spectral singularities which coexist with deep energy minima points of the transmission coefficient. The potential becomes invisible from left (or right) at different energy values. It is possible to make this potential bidirectional invisible at several energies by choosing appropriate values of the parameters in the potential.

In the other case $(d \rightarrow i d)$ we have penetrating as well as free states. Remarkably penetrating states are reciprocal for all energies even though the system is PT-symmetric non-Hermitian. No SS is present in this case. However MSS are shown to be present for the free states. Aspects of invisibility are also discussed for this case.

Now we present the plan of this paper. In Sec. 2 we discuss the nature of the real potential and complexify it in a PT-symmetric manner. In Sec. 3.1 we discuss the bound states of the non-Hermitian potential when $\mu \rightarrow i \mu$. Various aspects of scattering states for this case have been considered in Sec. 3.2. Sec. 4 is for the discussion of penetrating and free states for the second case $(d \rightarrow i d)$. Sec. 5 is reserved for discussions and conclusions.

\section{The Potential and PT-symmetrization}

The potential in Eq. (1) has a minima at $\mathrm{x}=0$ and asymptotically goes to a finite value $V_{0} e^{ \pm 2 \mu}$ as $x \rightarrow \pm \infty$. This potential exhibits bound states, reflective states and free states depending on the energy of the incident particle. The particle have bound states if its energy is between 0 to $V_{0} e^{-2 \mu}$ (region I in the Fig.1) and it oscillates back and fourth inside the potential. If the particle energy $\mathrm{E}$ is in between $V_{0} e^{-2 \mu}$ and $V_{0} e^{2 \mu}$ then we have reflecting states (region II in the Fig.1). Particle comes from left reflected back to $-\infty$ by the potential rise to the right. In the region III of the Fig.1 particle can move freely between $-\infty$ to $+\infty$ as its energy $E>V_{0} e^{2 \mu}$.

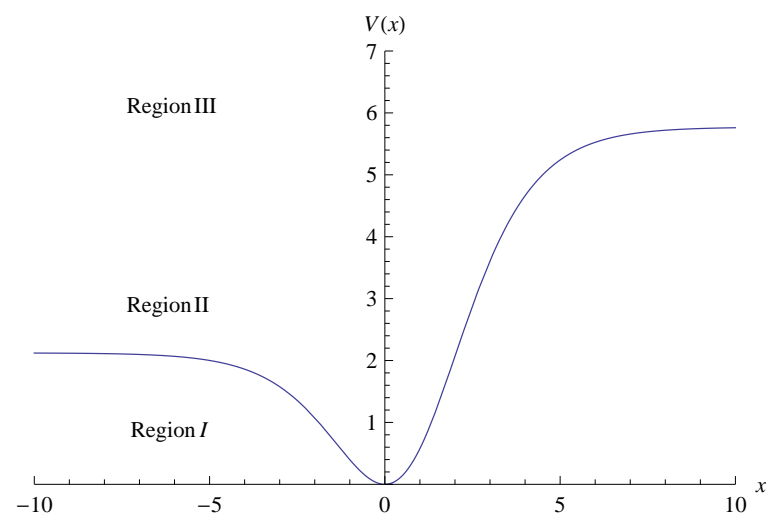

Fig.1: 1 -d real potential $\left(V_{0}=3.5, \mu=.25\right)$ which admits bound (Region-I), reflection 
(Region-II) and free states (Region-III).

This Hermitian potential in Eq. (1) can be complexified in many ways by considering one or more of the parameters $\mu, d$ and $V_{0}$ complex. However not all of them are PTsymmetric which are of our interest. In two simple ways this potential can be made PT-symmetric non-Hermitian;

Case-I. When $\mu$ is purely imaginary $(\mu \rightarrow i \mu)$, the potential becomes,

$$
V(x)=V_{0} \cosh ^{2}(i \mu)\{\tanh [(x-i \mu d) / d]+\tanh (i \mu)\}^{2}
$$

This potential is invariant under combined PT-transformation.

Case-II. On the other hand the potential in Eq. (1) turns out to be a PT-symmetric non-Hermitian if we substitute $d \rightarrow i d$ with a purely imaginary shift $\zeta$ (where $\zeta$ can have arbitrary values between $-\infty$ to $+\infty$ ) to the $\mathrm{x}$ coordinate and written as,

$$
V(x+i \zeta)=V_{0} \cosh ^{2} \mu\{\tanh [(x+i \zeta-\mu i d) / i d]+\tanh (\mu)\}^{2}
$$

In the first case we have entirely real bound states and PT-symmetry is unbroken for all values of the parameter. Scattering states show many important features. In the second case we have penetrating states and free states.

\section{Non-Hermitian PT-symmetric: case I $(\mu \rightarrow i \mu)$}

We begin the section with the general solution of the Schroedinger equation for the Hermitian potential in Eq. (1) which is given by,

$$
\psi(z)=\frac{e^{-a z}}{\left(e^{z}+e^{-z}\right)^{b}}\left[N F_{1}+M\left(\frac{e^{z}}{e^{z}+e^{-z}}\right)^{-(a+b)} F_{2}\right]
$$

where, $z=(x-\mu d) / d, v=\left(2 m d^{2} / \hbar^{2}\right) V_{0}, \epsilon=\left(2 m d^{2} / \hbar^{2}\right) E$, M and $\mathrm{N}$ are the normalization constants. $F_{1}$ and $F_{2}$ are the two solutions of hypergeometric equation,

$$
\begin{aligned}
& F_{1}=F\left(b+1 / 2-\sqrt{v \cosh ^{2} \mu+1 / 4}, b+1 / 2+\sqrt{v \cosh ^{2} \mu+1 / 4} ;|a+b+1| ; \frac{e^{-z}}{e^{z}+e^{-z}}\right) \\
& F_{2}=F\left(-a+1 / 2-\sqrt{v \cosh ^{2} \mu+1 / 4},-a+1 / 2+\sqrt{v \cosh ^{2} \mu+1 / 4} ;|-a-b+1| ; \frac{e^{-z}}{e^{z}+e^{-z}}\right)
\end{aligned}
$$

where the parameters $a$ and $b$ are defined as,

$$
a^{2}+b^{2}=-\epsilon+v \cosh 2 \mu ; 2 a b=v \sinh 2 \mu .
$$


Eq. (6) further can be expressed as,

$$
\begin{aligned}
a & =\frac{1}{2} \sqrt{v e^{2 \mu}-\epsilon}-\frac{1}{2} \sqrt{v e^{-2 \mu}-\epsilon} \equiv \frac{1}{2} \kappa_{+}-\frac{1}{2} \kappa_{-} ; \\
b & =\frac{1}{2} \sqrt{v e^{2 \mu}-\epsilon}+\frac{1}{2} \sqrt{v e^{-2 \mu}-\epsilon} \equiv \frac{1}{2} \kappa_{+}+\frac{1}{2} \kappa_{-} ;
\end{aligned}
$$

As for bound states $\epsilon$ is always less than $v e^{ \pm 2 \mu}, \kappa_{+}$and $\kappa_{-}$are real for the Hermitian case. The solution of the Schroedinger equation which is finite at $z=\infty$ is therefore obtained from the general solutions in Eq. (4) as,

$$
\begin{gathered}
\psi=\frac{e^{-a z}}{\left(e^{z}+e^{-z}\right)^{b}} N F_{1}=\frac{N e^{-a z}}{\left(e^{z}+e^{-z}\right)^{b}} F\left(b+1 / 2-\sqrt{v \cosh ^{2} \mu+1 / 4},\right. \\
\left.b+1 / 2+\sqrt{v \cosh ^{2} \mu+1 / 4} ;|a+b+1| ; \frac{e^{-z}}{e^{z}+e^{-z}}\right)
\end{gathered}
$$

which has the following limiting behavior at $z \rightarrow-\infty$,

$$
\begin{array}{r}
\psi \rightarrow \frac{\Gamma(a+b+1) \Gamma(b-a) e^{(a-b) z}}{\Gamma\left(b+1 / 2-\sqrt{v \cosh ^{2} \mu+1 / 4}\right) \Gamma\left(b+1 / 2+\sqrt{v \cosh ^{2} \mu+1 / 4}\right)} \\
+\frac{\Gamma(a+b+1) \Gamma(a-b) e^{(b-a) z}}{\Gamma\left(a+1 / 2-\sqrt{v \cosh ^{2} \mu+1 / 4}\right) \Gamma\left(a+1 / 2+\sqrt{v \cosh ^{2} \mu+1 / 4}\right)}
\end{array}
$$

The allowed bound states are obtained for the finite asymptotic wavefunction depending on the situations $a>b$ or $b>a$.

Now with this general discussion on the Hermitian potential we are ready to take up the non-Hermitian extensions of this potential.

\subsection{Bound States}

In this case we denote, $z=(x-i \mu d) / d$ and re-express $a$ and $b$ by using Eq. (6) for an imaginary $\mu$ as,

$$
\begin{aligned}
a^{I} & =\frac{1}{2} \sqrt{v e^{2 i \mu}-\epsilon}-\frac{1}{2} \sqrt{v e^{-2 i \mu}-\epsilon} \equiv \frac{1}{2} k_{+}-\frac{1}{2} k_{-} \\
b^{I} & =\frac{1}{2} \sqrt{v e^{2 i \mu}-\epsilon}+\frac{1}{2} \sqrt{v e^{-2 i \mu}-\epsilon} \equiv \frac{1}{2} k_{+}+\frac{1}{2} k_{-} .
\end{aligned}
$$


From the above equation one can easily see that $a^{I}$ becomes purely imaginary and on the other hand $b^{I}$ is still real. The general solution for the Schroedinger equation for this non-Hermitian PT-symmetric potential in Eq. (2) now is written (with $a^{I} \rightarrow i a^{\prime}$ ),

$$
\psi^{I}(z)=\frac{N e^{-i a^{\prime} z}}{\left(e^{z}+e^{-z}\right)^{b^{I}}} F_{1}^{I}+M\left(\frac{e^{z}}{e^{z}+e^{-z}}\right)^{-\left(i a^{\prime}+b^{I}\right)} \frac{e^{i a^{\prime} z}}{\left(e^{z}+e^{-z}\right)^{b^{I}}} F_{2}^{I}
$$

where $F_{1}^{I}$ and $F_{2}^{I}$ are obtained from Eq. (5) by substituting $a, b$ and $z$ for the case of imaginary $\mu$. The finite wavefunction at $z \rightarrow+\infty$ for the bound states is,

$$
\begin{aligned}
& \psi^{I}(z)= \frac{N e^{-i a^{\prime} z}}{\left(e^{z}+e^{-z}\right)^{b^{I}}} F\left(b^{I}+1 / 2-\sqrt{v \cosh ^{2}(i \mu)+1 / 4},\right. \\
&\left.b^{I}+1 / 2+\sqrt{v \cosh ^{2}(i \mu)+1 / 4} ;\left|i a^{\prime}+b^{I}+1\right| ; \frac{e^{-z}}{e^{z}+e^{-z}}\right)
\end{aligned}
$$

The above wave function has the following limiting behavior at $z \rightarrow-\infty$,

$$
\begin{aligned}
\psi^{I}(z) \rightarrow & \frac{\Gamma\left(i a^{\prime}+b^{I}+1\right) \Gamma\left(b^{I}-i a^{\prime}\right) e^{i a^{\prime} z} e^{-b^{I} z}}{\Gamma\left(b^{I}+1 / 2-\sqrt{v \cosh ^{2}(i \mu)+1 / 4}\right) \Gamma\left(b^{I}+1 / 2+\sqrt{v \cosh ^{2}(i \mu)+1 / 4}\right)} \\
& +\frac{\Gamma\left(i a^{\prime}+b^{I}+1\right) \Gamma\left(i a^{\prime}-b^{I}\right) e^{-i a^{\prime} z} e^{b^{I} z}}{\Gamma\left(i a^{\prime}+1 / 2-\sqrt{v \cosh ^{2}(i \mu)+1 / 4}\right) \Gamma\left(i a^{\prime}+1 / 2+\sqrt{v \cosh ^{2}(i \mu)+1 / 4}\right)}
\end{aligned}
$$

For $b^{I}>0$, to have a finite wavefunction at $z \rightarrow-\infty$ the argument of one of the Gamma function in the denominator of the first term must be a negative integer. This fixes the allowed ranges of energy for the bound states where,

$$
b^{I}=b_{n}^{I}=\sqrt{v \cos ^{2} \mu+1 / 4}-(n+1 / 2) ; \quad a^{I}=a_{n}^{I}=\frac{i v \sin 2 \mu}{2\left(\sqrt{v \cos ^{2} \mu+1 / 4}-(n+1 / 2)\right)} .
$$

The bound state energy for this case is then $\epsilon_{n}^{I}=v \cos (2 \mu)-\left(a^{I}\right)^{2}-\left(b^{I}\right)^{2}$,

$$
\epsilon_{n}^{I}=v \cos (2 \mu)+\frac{v^{2} \sin ^{2} 2 \mu}{4\left(\sqrt{v \cos ^{2} \mu+1 / 4}-(n+1 / 2)\right)^{2}}-\left[\sqrt{v \cos ^{2} \mu+1 / 4}-(n+1 / 2)\right]^{2}
$$

where, $n=0,1,2 \ldots<\left(\sqrt{v \cos ^{2} \mu+1 / 4}-1 / 2\right)$, and the corresponding wave function is,

$$
\psi_{n}^{I}=\frac{N e^{-a_{n}^{I} z}}{\left(e^{z}+e^{-z}\right)^{b_{n}^{I}}} F\left(-n, 2 \sqrt{v \cos ^{2} \mu+1 / 4}-n ;\left|a_{n}^{I}+b_{n}^{I}+1\right| ; \frac{e^{-z}}{e^{z}+e^{-z}}\right) .
$$


Now to have a PT-unbroken phase the wavefunction need to satisfy the condition $P T \psi=$ $A \psi$. The PT-transformation changes $a_{n} \rightarrow-a_{n}^{I}, z \rightarrow-z, b_{n} \rightarrow b_{n}^{I}, v \rightarrow v$ and hence we obtain,

$$
P T \psi_{n}^{I}=\frac{N e^{-a_{n}^{I} z}}{\left(e^{z}+e^{-z}\right)^{b_{n}^{I}}} F\left(-n, 2 \sqrt{v \cos ^{2} \mu+1 / 4}-n ;\left|-a_{n}^{I}+b_{n}^{I}+1\right| ; \frac{e^{z}}{e^{z}+e^{-z}}\right)
$$

Using the standard properties of hypergeometric functions we obtain $P T \psi_{n}^{I}$ for the case of bound states as,

$$
\begin{aligned}
P T \psi_{n}^{I}= & \frac{\Gamma\left(a_{n}^{I}+b_{n}^{I}+1\right) \Gamma\left(a_{n}^{I}-b_{n}^{I}\right)}{\Gamma\left(a_{n}^{I}+b_{n}^{I}+n+1\right) \Gamma\left(a_{n}^{I}-b_{n}^{I}-n\right)}\left(\frac{N e^{-a_{n}^{I} z}}{\left(e^{z}+e^{-z}\right)^{b_{n}^{I}}}\right) \\
& F\left(-n, 2 \sqrt{v \cos ^{2} \mu+1 / 4}-n ;\left|a_{n}^{I}+b_{n}^{I}+1\right| ; \frac{e^{-z}}{e^{z}+e^{-z}}\right)=A_{n} \psi_{n}^{I}
\end{aligned}
$$

where,

$$
A_{n}=\frac{\Gamma\left(a_{n}^{I}+b_{n}^{I}+1\right) \Gamma\left(a_{n}^{I}-b_{n}^{I}\right)}{\Gamma\left(a_{n}^{I}+b_{n}^{I}+n+1\right) \Gamma\left(a_{n}^{I}-b_{n}^{I}-n\right)} \quad \text { is a pure number for the n-th state. }
$$

In this case the entire bound state spectrum is in PT-unbroken phase and the energy eigenvalues in Eq. (15) is always real. Similar result can also be obtained for $b^{I}<0$.

\subsection{Scattering States and Spectral Properties}

Now for the scattering states we have to consider all the solutions of Schroedinger equation for the PT symmetric potential in Eq. (2),

$$
\begin{aligned}
\psi_{1}(z)= & N e^{\frac{1}{2} i\left(k_{+}-k_{-}\right) z}\left(e^{z}+e^{-z}\right)^{\frac{1}{2} i\left(k_{+}+k_{-}\right) z} \\
& F\left(-\frac{1}{2} i k_{+}-\frac{1}{2} i k_{-}+\frac{1}{2}-\gamma,-\frac{1}{2} i k_{+}-\frac{1}{2} i k_{-}+\frac{1}{2}+\gamma ;\left|1-i k_{+}\right| ; \frac{e^{-z}}{e^{z}+e^{-z}}\right) \\
\psi_{2}(z)= & N e^{\frac{1}{2} i\left(k_{+}-k_{-}\right) z}\left(e^{z}+e^{-z}\right)^{\frac{1}{2} i\left(k_{+}+k_{-}\right) z} \cdot\left(\frac{e^{-z}}{e^{z}+e^{-z}}\right) \\
& F\left(\frac{1}{2} i k_{+}-\frac{1}{2} i k_{-}+\frac{1}{2}-\gamma, \frac{1}{2} i k_{+}-\frac{1}{2} i k_{-}+\frac{1}{2}+\gamma ;\left|1+i k_{+}\right| ; \frac{e^{-z}}{e^{z}+e^{-z}}\right) .
\end{aligned}
$$

For the case of scattering

$$
k_{+}=\sqrt{\epsilon-v e^{2 i \mu}} ; \quad k_{-}=\sqrt{\epsilon-v e^{2 i \mu}}
$$

and the general scattering wavefunction is written as,

$$
\psi_{\text {scatt }}(z)=A \psi_{1}(z)+B \psi_{2}(z)
$$


where $A$ and $B$ are the arbitrary constants. By considering the asymptotic behavior of $\psi_{1}$ and $\psi_{2}$ and using the method outlined in Ref. [37] we construct the left and right handed transmission and reflection amplitudes as,

$$
\begin{gathered}
t_{l}=\frac{1}{G 2} ; \quad r_{l}=\frac{G 1}{G 2}, \\
t_{r}=\frac{G 2 . G 3-G 1 . G 4}{G 2} ; \quad r_{r}=-\frac{G 4}{G 2} .
\end{gathered}
$$

where G1, G2 G3, G4 are calculated in terms of Gamma functions as,

$$
\begin{aligned}
G 1 & =\frac{\Gamma\left(1-i k_{+}\right) \Gamma\left(i k_{-}\right)}{\Gamma\left(-\frac{1}{2} i k_{+}+\frac{1}{2} i k_{-}+\frac{1}{2}+\gamma\right) \Gamma\left(-\frac{1}{2} i k_{+}+\frac{1}{2} i k_{-}+\frac{1}{2}-\gamma\right)} ; \\
G 2 & =\frac{\Gamma\left(1-i k_{+}\right) \Gamma\left(-i k_{-}\right)}{\Gamma\left(-\frac{1}{2} i k_{+}-\frac{1}{2} i k_{-}+\frac{1}{2}-\gamma\right) \Gamma\left(-\frac{1}{2} i k_{+}-\frac{1}{2} i k_{-}+\frac{1}{2}+\gamma\right)} ; \\
G 3 & =\frac{\Gamma\left(1+i k_{+}\right) \Gamma\left(i k_{-}\right)}{\Gamma\left(\frac{1}{2} i k_{+}+\frac{1}{2} i k_{-}+\frac{1}{2}+\gamma\right) \Gamma\left(\frac{1}{2} i k_{+}+\frac{1}{2} i k_{-}+\frac{1}{2}-\gamma\right)} \\
G 4 & =\frac{\Gamma\left(1+i k_{+}\right) \Gamma\left(-i k_{-}\right)}{\Gamma\left(\frac{1}{2} i k_{+}-\frac{1}{2} i k_{-}+\frac{1}{2}-\gamma\right) \Gamma\left(\frac{1}{2} i k_{+}-\frac{1}{2} i k_{-}+\frac{1}{2}+\gamma\right)}
\end{aligned}
$$

Now we have the following interesting observations for this non-Hermitian PT- symmetric system.

\subsubsection{Reciprocity and Handedness}

It is clear from Eq. (22), (23) and (24) that $r_{l} \neq r_{r}$ for both real or complex potential. For the Hermitian case $k_{+}, k_{-}$are real and it is straight forward to verify that

$$
R_{l} \equiv\left|r_{l}\right|^{2}=\frac{\left(G 1^{*}\right) \cdot(G 1)}{|G 2|^{2}}=\frac{(G 4) \cdot\left(G 4^{*}\right)}{|G 2|^{2}}=\left|r_{r}\right|^{2} \equiv R_{r} \text { and } T_{l}=T_{r}
$$

Thus we have reciprocity in the case of scattering from a real potential. For the PT-symmetric non-Hermitian case $k_{+}, k_{-}$are complex with the properties $k_{+}^{*}=k_{-}$and $k_{-}^{*}=k_{+}$, hence $R_{l} \neq R_{r}$ even though $T_{l}=T_{r}$. This is quite natural as parity is violated in the case of PT-symmetric potential.

We show that at some particular positive energies $R_{l}=R_{r}$ even for the non-Hermitian PT-symmetric potential. In fact for any parametric range of $\mathrm{v}$ and $\mu$ (except $\mu=n \pi$, $\mathrm{n}=0,1 / 2,1,3 / 2, \ldots$ when the potential become either real or indefinite) we find certain discrete energy points where $R_{l}=R_{r}$. Thus reciprocity is restored even for PT-symmetric non-Hermitian potential at some discrete energies. It is clear from the Eq. (22) to (24) that reciprocity is restored when $|G 1|^{2}=|G 4|^{2}$ for the PT-symmetric non-Hermitian case. This result is well illustrated with the help of Fig.2 where reciprocity is obtained in 
one or multiple energy values.
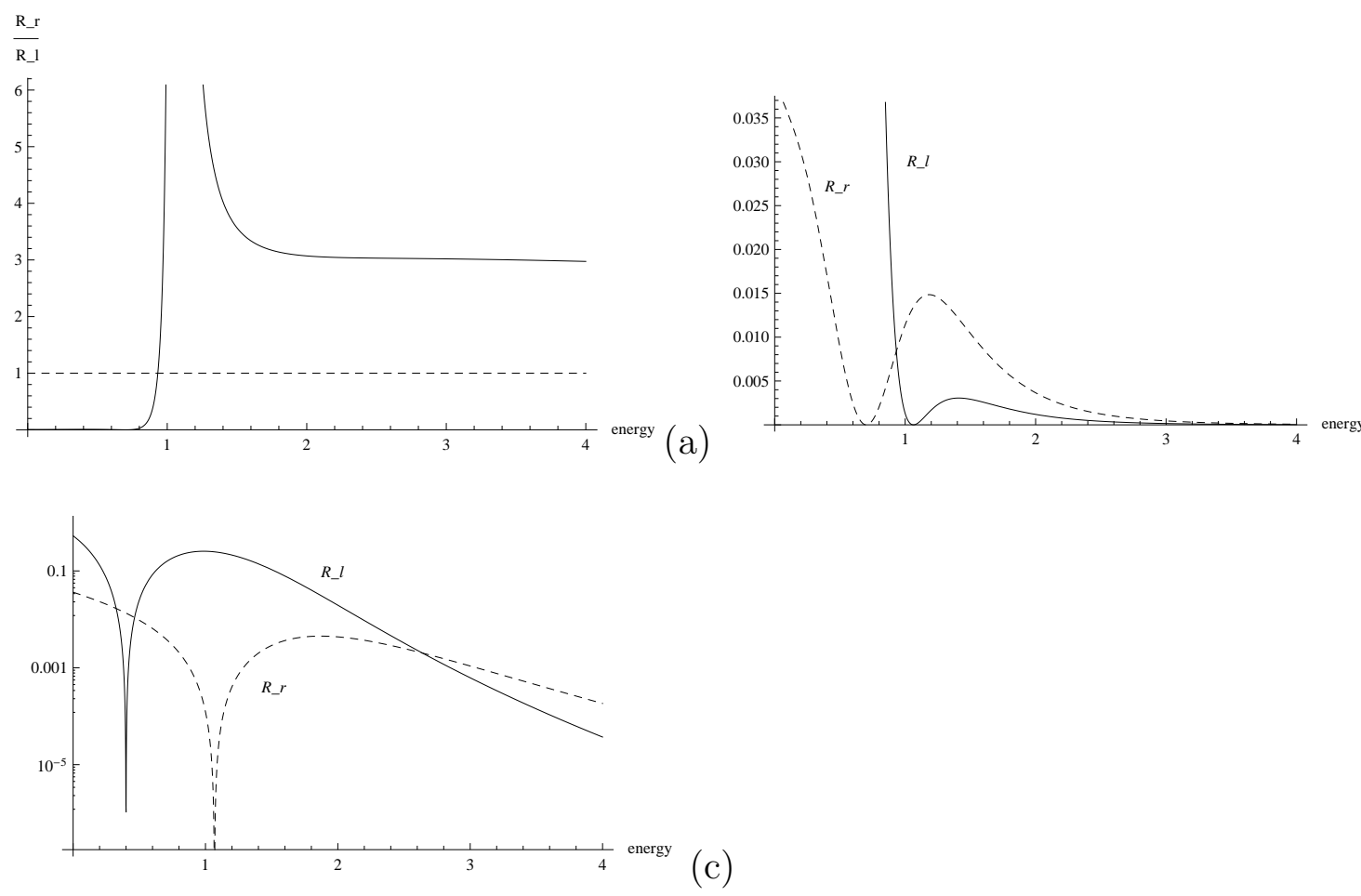

Fig.2: Shows different points where reciprocity is restored even for PT-symmetric nonHermitian system. We have one energy point where $R_{l}=R_{r}$ for $v=1$ and $\mu=\pi / 12$, in Fig.2(a). Fig.2(b) shows the left (solid line) and right (dashed line) handed reflection coefficients are equal at the energy point E=0.9943. In Fig.2(c) we have obtained three different energy points 0.3255, 0.4642 and 2.639 where reciprocity is restored.

\subsubsection{Multiple Spectral Singularities (MSS)}

SS are the obstacles for the development of a consistent quantum theory with nonHermitian potential and need to be located with extensive care. We have seen at most one $\mathrm{SS}$ in the most of the PT-symmetric non-Hermitian systems studied in literature so far $[26,27,29]$. We show the existence of MSS in this PT-symmetric non-Hermitian system. $\mathrm{SS}$ arise due to the divergence of the Gamma functions in the numerator of the reflection and transmission coefficients. The divergences occur in $R_{l}\left(=\left|r_{l}\right|^{2}\right)$ from Eq. (22) due to the Gamma functions in the numerator which diverges at multiple values of positive energy. This exciting result is demonstrated in Fig.3.

We observe another interesting feature of this PT-symmetric non-Hermitian potential. The transmission coefficient $T$ oscillates with deep energy minima at the same positive energy points where $R_{l}$ diverges. We note that divergence of the reflection coefficient 
is dominated over these deep minima leading to an overall divergence of $R_{l}+T$ in these energy points. The coexistence of deep energy minima of $T$ at these MSS points is a unique feature of this PT-symmetric non-Hermitian potential. We further observe that unitarity is restored at certain discrete energy values (Fig.3 (b)) and unitarity is violated maximally at the spectral singular points. Unitarity is preserved at sufficient high energy.

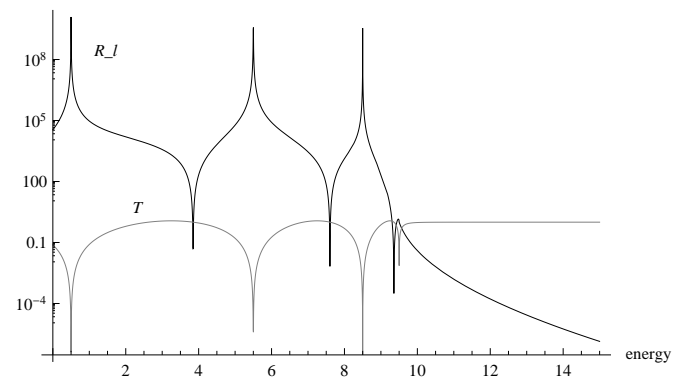

(a)

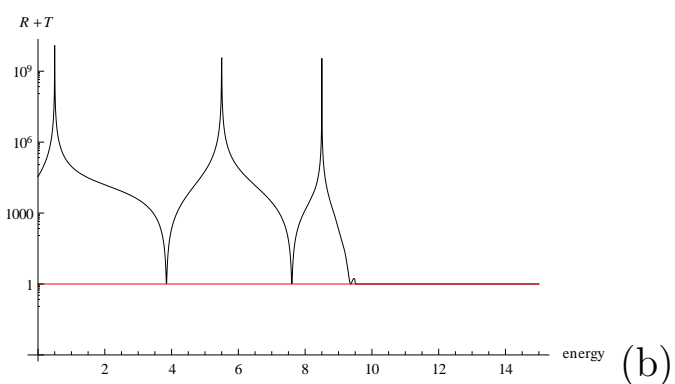

Fig.3: The existence of MSS in this non-Hermitian model is shown. In Fig.3(a) ( $v=9.5, \mu=6.2832$ ) the coexistence of divergences of $R_{l}$ (dark line) and deep energy minimas of $T$ (gray line) are shown. Fig.3(b) shows the multiple spectral singular points where $R_{l}+T$ diverges and in the other hand it also shows the points where unitarity is preserved $\left(R_{l}+T=1\right)$.

\subsubsection{Invisibility}

In this section we show that this PT-symmetric non-Hermitian potential has yet another interesting property of scattering. The potential become reflectionless for both left and right incidents for certain parametric values. However we would like to emphasize that the energy values at which the potential become reflectionless is different for left and right incidence i.e. $\mathrm{E}\left(R_{l}=0\right) \neq \mathrm{E}\left(R_{r}=0\right)$. Further we observe that the transmission coefficient becomes unity at these point. This implies the potential becomes invisible from both sides. However left invisible point is different from right invisible point for this potential. By choosing the appropriate values of the parameters in the potential it is possible to make this potential bidirectional invisible at multiple values of energies. All these features are demonstrated in Fig.4. 


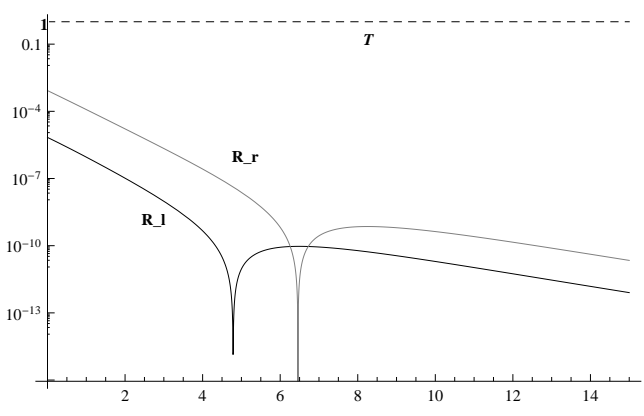

(a)
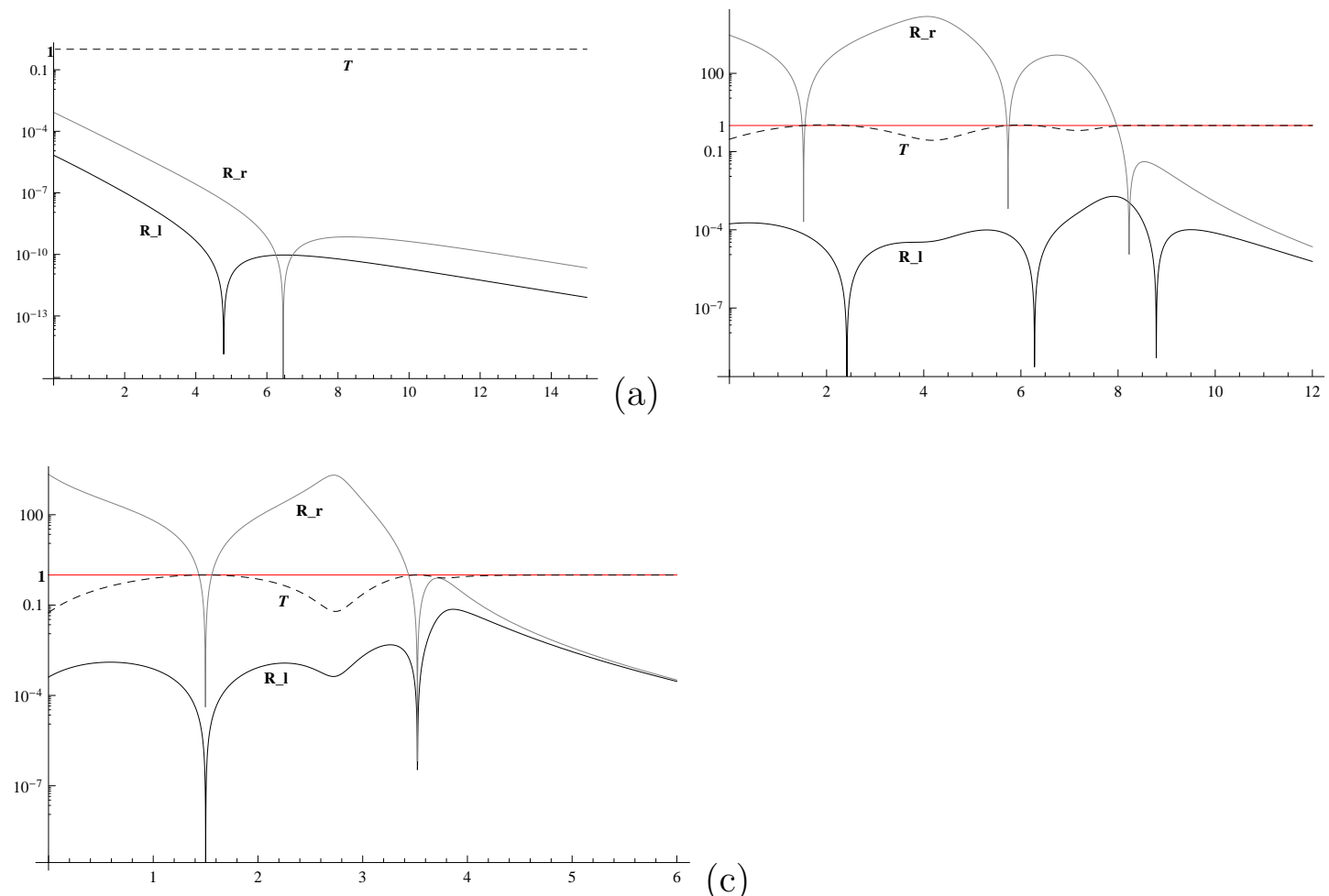

(c)

Fig.4: Left (solid line) and right (gray line) handed $R$ and $T$ (dashed line) are plotted against energy to show the invisibility $(R=0, T=1)$ of the potential at discrete energies; In Fig. 4.(a) for $v=3.54, \mu=1.11$ invisible points occurs at different energies for $R_{l}$ (at $E=4.788$ ) and $R_{r}$ (at $\left.E=6.455\right)$. Fig.4(b) shows multiple invisible points for $v=8.24, \mu=$ 6.24 which are unidirectional in nature. Multiple bidirectional invisible points are shown in Fig.4(c) for specific values of parameters $v=3.75 ; \mu=3.12$ where $R_{l}=R_{r}=0$, and $T=1$ for the same energies.

\section{Non-Hermitian PT-symmetric: case-II $(d \rightarrow i d)$}

Time independent Schroedinger equation (TISE) for this case is written as,

$$
\frac{\hbar^{2}}{2 m d^{2}} \frac{d^{2} \psi}{d z^{2}}+V(z) \psi=E \psi
$$

where $\mathrm{z}$ is taken as $z=-i X / d-\mu$, with $X=x+i \zeta$. Note that the differential term in this equation comes with wrong sign due to the presence of $d^{2}$ term. However this equation can be interpreted as TISE for a upside down potential of the original one with energy eigenvalues (-E). This upside down potential in the real plane looks as, 


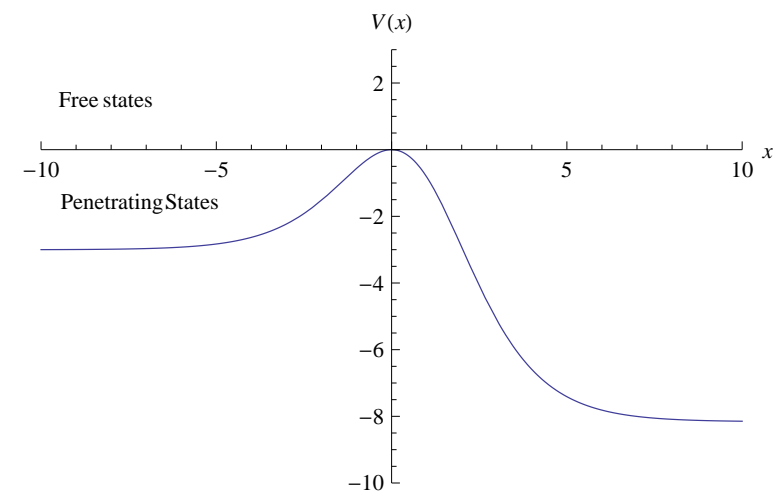

Fig.5: The effective potential (upside down of the original real potential) for the case $d \rightarrow i d$ is plotted for $V_{0}=-4.95, \mu=.25$ and $d=2.50$.

It behaves like a potential barrier with a maxima at $\mathrm{x}=0$. We have penetrating states if the particle has negative energy with magnitude less than $v e^{-2 \mu}$. On the other hand it accepts free states when the energy of the particle is more than the barrier height.

The penetrating state solutions are given by,

$$
\begin{aligned}
\psi_{1}(z)= & N e^{-a z}\left(e^{z}+e^{-z}\right)^{-b z} F\left(b+\frac{1}{2}-\sqrt{-v \cosh ^{2}(\mu)+1 / 4}\right. \\
& \left.b+\frac{1}{2}+\sqrt{-v \cosh ^{2}(\mu)+1 / 4} ;|a+b+1| ; \frac{e^{-z}}{e^{z}+e^{-z}}\right) \\
\psi_{2}(z)= & N e^{b z}\left(e^{z}+e^{-z}\right)^{a z} F\left(-a+\frac{1}{2}-\sqrt{-v \cosh ^{2}(\mu)+1 / 4}\right. \\
& \left.-a+\frac{1}{2}+\sqrt{-v \cosh ^{2}(\mu)+1 / 4} ;|1-a-b| ; \frac{e^{-z}}{e^{z}+e^{-z}}\right)
\end{aligned}
$$

with

$$
a^{2}+b^{2}=-\epsilon-v \cosh 2 \mu ; \quad 2 a b=-v \sinh 2 \mu ;
$$

and $k_{+}=\sqrt{\epsilon+v e^{2 \mu}}, k_{-}=\sqrt{\epsilon+v e^{-2 \mu}}$ so that

$$
a=-1 / 2\left(i k_{+}+i k_{-}\right) ; \quad b=1 / 2\left(-i k_{+}+i k_{-}\right) .
$$

The wavefunction for this penetrating state is written in a general form as,

$$
\psi(z)=A \psi_{1}(z)+B \psi_{2}(z)
$$

We calculate the left and right handed penetrating amplitudes from the asymptotic behavior of Eq. (30) as,

$$
\begin{gathered}
t_{l}=\frac{1}{P 2} ; \quad r_{l}=\frac{P 1}{P 2}, \\
t_{r}=\frac{P 2 . P 3-P 1 . P 4}{P 2} ; \quad r_{r}=-\frac{P 4}{P 2} .
\end{gathered}
$$


where P1, P2, P3, P4 are expressed in terms of Gamma function written as,

$$
\begin{aligned}
P 1 & =\frac{\Gamma\left(1-i k_{+}\right) \Gamma\left(-i k_{-}\right)}{\Gamma\left(-\frac{1}{2} i k_{+}-\frac{1}{2} i k_{-}+\frac{1}{2}+\gamma^{\prime}\right) \Gamma\left(-\frac{1}{2} i k_{+}-\frac{1}{2} i k_{-}+\frac{1}{2}-\gamma^{\prime}\right)} \\
P 2 & =\frac{\Gamma\left(1-i k_{+}\right) \Gamma\left(i k_{-}\right)}{\Gamma\left(-\frac{1}{2} i k_{+}+\frac{1}{2} i k_{-}+\frac{1}{2}-\gamma^{\prime}\right) \Gamma\left(-\frac{1}{2} i k_{+}+\frac{1}{2} i k_{-}+\frac{1}{2}+\gamma^{\prime}\right)} \\
P 3 & =\frac{\Gamma\left(1+i k_{+}\right) \Gamma\left(-i k_{-}\right)}{\Gamma\left(\frac{1}{2} i k_{+}-\frac{1}{2} i k_{-}+\frac{1}{2}+\gamma^{\prime}\right) \Gamma\left(\frac{1}{2} i k_{+}-\frac{1}{2} i k_{-}+\frac{1}{2}-\gamma^{\prime}\right)} \\
P 4 & =\frac{\Gamma\left(1+i k_{+}\right) \Gamma\left(i k_{-}\right)}{\Gamma\left(\frac{1}{2} i k_{+}+\frac{1}{2} i k_{-}+\frac{1}{2}-\gamma^{\prime}\right) \Gamma\left(\frac{1}{2} i k_{+}+\frac{1}{2} i k_{-}+\frac{1}{2}+\gamma^{\prime}\right)}
\end{aligned}
$$

where $\gamma^{\prime}=\sqrt{-v \cosh ^{2}(\mu)+1 / 4}$. Note $P 1^{*}=P 4 ; \quad P 2^{*}=P 3$, as $k_{+}$and $k_{-}$are all real quantities in this case. We observe,

$$
r_{l} \neq r_{r} ; \text { but } R_{l} \equiv\left|r_{l}\right|^{2}=\left|r_{r}\right|^{2} \equiv R_{r} \text { and } T_{l}=T_{r} \text {. }
$$

This implies we have reciprocity for this non-Hermitian PT-symmetric system for all values of energy. However as expected unitarity is violated, i.e $R+T \neq 1$ (both for left and right handed cases) for this model. We further observe no SS is present for penetrating states and the potential never becomes reflectionless.

\subsection{Free states and Spectral Properties}

We have free state solutions for this case when the energy of the incident particle is positive. So the free state solutions of TISE are alike the solutions given in Eq. (27) with

$$
a=1 / 2\left(k_{+}-k_{-}\right) ; \quad b=1 / 2\left(k_{+}+k_{-}\right) ;
$$

where $k_{+}=\sqrt{\epsilon-v e^{2 \mu}}$ and $k_{-}=\sqrt{\epsilon-v e^{-2 \mu}}$ for all positive energy of the incident particle. The scattering wavefunction is then expressed as,

$$
\begin{aligned}
\psi(z)= & \left(e^{-\frac{1}{2}\left(k_{+}-k_{-}\right) z}\left(e^{z}+e^{-z}\right)^{-\frac{1}{2}\left(k_{+}+k_{-}\right) z}\right)\left[A F \left(\frac{1}{2} k_{+}+\frac{1}{2} k_{-}+\frac{1}{2}-\gamma,\right.\right. \\
& \left.\frac{1}{2} k_{+}+\frac{1}{2} k_{-}+\frac{1}{2}+\gamma ;\left|1+k_{+}\right| ; \frac{e^{-z}}{e^{z}+e^{-z}}\right)+B\left(\frac{e^{-z}}{e^{z}+e^{-z}}\right)^{-k_{+}} \cdot \\
& \left.F\left(-\frac{1}{2} k_{+}+\frac{1}{2} i k_{-}+\frac{1}{2}-\gamma,-\frac{1}{2} i k_{+}+\frac{1}{2} i k_{-}+\frac{1}{2}+\gamma ;\left|1-k_{+}\right| ; \frac{e^{-z}}{e^{z}+e^{-z}}\right)\right]
\end{aligned}
$$

with $z=\frac{x+i \zeta}{i d}-\mu$. Now by considering the asymptotic behavior of the above scattering wavefunction at $x, \zeta \rightarrow \pm \infty$ we calculate the left and right handed scattering amplitudes as,

$$
t_{l}=\frac{1}{H 2} ; \quad r_{l}=\frac{H 1}{H 2}
$$




$$
t_{r}=\frac{H 2 . H 3-H 1 . H 4}{H 2} ; \quad r_{r}=-\frac{H 4}{H 2} .
$$

where $\mathrm{H} 1, \mathrm{H} 2, \mathrm{H} 3, \mathrm{H} 4$ are in terms of Gamma function as,

$$
\begin{aligned}
H 1 & =\frac{\Gamma\left(1+k_{+}\right) \Gamma\left(-k_{-}\right)}{\Gamma\left(\frac{1}{2} k_{+}-\frac{1}{2} k_{-}+\frac{1}{2}+\gamma\right) \Gamma\left(\frac{1}{2} k_{+}-\frac{1}{2} k_{-}+\frac{1}{2}-\gamma\right)} \\
H 2 & =\frac{\Gamma\left(1+k_{+}\right) \Gamma\left(k_{-}\right)}{\Gamma\left(\frac{1}{2} k_{+}+\frac{1}{2} k_{-}+\frac{1}{2}-\gamma\right) \Gamma\left(\frac{1}{2} k_{+}+\frac{1}{2} k_{-}+\frac{1}{2}+\gamma\right)} \\
H 3 & =\frac{\Gamma\left(1-k_{+}\right) \Gamma\left(-k_{-}\right)}{\Gamma\left(-\frac{1}{2} k_{+}-\frac{1}{2} k_{-}+\frac{1}{2}+\gamma\right) \Gamma\left(-\frac{1}{2} k_{+}-\frac{1}{2} k_{-}+\frac{1}{2}-\gamma\right)} \\
H 4 & =\frac{\Gamma\left(1-k_{+}\right) \Gamma\left(k_{-}\right)}{\Gamma\left(-\frac{1}{2} k_{+}+\frac{1}{2} k_{-}+\frac{1}{2}-\gamma\right) \Gamma\left(-\frac{1}{2} k_{+}+\frac{1}{2} k_{-}+\frac{1}{2}+\gamma\right)}
\end{aligned}
$$

We observe several interesting features in this case. In one hand $R_{l}$ diverges at several positive energy values leading to the existence of MSS. On the other hand the potential becomes reflectionless for the left in a different discrete energy as $R_{l}=0$. Since at the same energy point the transmission coefficient $T$ becomes unity the potential becomes invisible from left at that discrete energy.
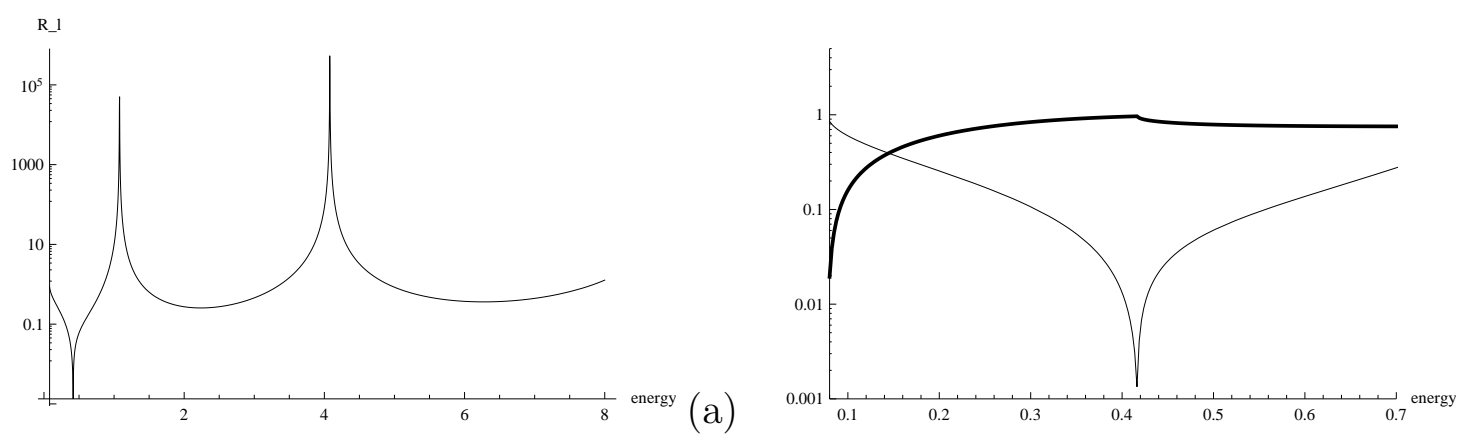

Fig.6: In Fig.6(a) spectral singular points are shown where left handed $R$ diverges $O\left(10^{6}\right)$ at more than one energy points for specific values of $v$ and $\mu(v=0.18, \mu=0.42)$. Reflectionless point at $E=0.4168$ is focused in Fig.6(b) where left handed $R$ (dark line) goes to zero and $T$ (bold line) is unity at that energy point.

Interestingly we observe no invisible point when the particle incidents from right. $R_{r}$ never becomes zero for this model as none of the argument of the Gamma functions in denominator of $R_{r}$ becomes zero or negative integer. So the potential in this PTsymmetric configuration shows only unidirectional invisibility for any parametric regime. 


\section{Conclusions and Discussions}

We have complexified a one dimensional potential which exhibit bound, reflecting and free states to capture various interesting properties of non-Hermitian theories. It leads to PT-symmetric non-Hermitian system when it is complexified by changing (i) $\mu \rightarrow i \mu$ and (ii) $d \rightarrow i d$. In the first case, when we consider bound states the system is always in PT-unbroken phase leading to the entire real spectrum. We further calculate reflection and transmission amplitudes for both left and right incident particles by considering the asymptotic behavior of the scattering states. The reflection amplitudes are different for left and right incidence both for real as well as PT-symmetric non-Hermitian systems (case-I). This implies no-reciprocity for non-Hermitian PT-symmetric system. This is natural as reciprocity is a property of a system which remains invariant under the space inversion (parity). PT-symmetric non-Hermitian potentials essentially change under parity. However for real potential $R_{l}=\left|r_{l}\right|^{2}=\left|r_{r}\right|^{2}=R_{r}$ is always true and we have reciprocity. We have shown that this particular non-Hermitian PT-symmetric system is reciprocal at certain discrete energies as shown in Fig.2.

The most interesting and new feature in the scattering of this PT-symmetric nonHermitian system is the existence of multiple spectral singularities. This has been shown by considering the divergence of $R$ at different positive energies. On the other hand transmission coefficient $T$ has deep energy minima at these SS points. Coexistence of MSS with deep energy minima of transmission coefficient is a unique characteristic of this system. We have further shown that the potential becomes invisible $(R=0, T=1)$ at certain energies. It is possible to make this potential bidirectional invisible at multiple positive values of energy by adjusting the parameter of the potential

In the second case $(d \rightarrow i d)$ the system provides penetrating states if the energy of the particle is less than zero and greater than $-v e^{-2 \mu}$. We have calculated the different transmission and reflection amplitudes both for left and right incidence. We have analytically shown the reciprocity for this PT-symmetric non-Hermitian system. No SS or reflectionless conditions are present for the penetrating states. We have also constructed the free states for this case where the energy of the particle is positive. We have found the MSS points for this case and the system becomes reflectionless at certain energies. It will be interesting to restore both reciprocity and unitarity for non-Hermitian PT-symmetric system at certain energies.

\section{References}

[1] C. M. Bender and S. Boettcher Phys.Rev.Lett. 80, 5243 (1998).

[2] A. Mostafazadeh, Int. J. Geom. Meth. Mod. Phys. 7, 1191(2010) and references therein.

[3] C. M. Bender, Rep.Prog. Phys. 70, 947 (2007) and references therein. 
[4] C. M. Bender, D. C. Brody, J. Caldeira, B. K. Meister, arXiv 1011.1871 (2010).

[5] S. Longhi Phys. Rev. B 80, 165125 (2009).

[6] B. F. Samsonov J. Phys. A 43, 402006 (2010); Math. J. Phys. A: Math. Gen. 38, L571 (2005).

[7] P. E.G. Assis, A. Fring, Journal of Physics A: Math. Theor., 42, 105206 (2009); A. Fring, Phil. Trans. R. Soc. A, 37120120046 (2013).

[8] B. Basu-Mallick, Int. J. of Mod. Phys. B 16, 1875 (2002); B. Basu-Mallick, T. Bhattacharyya, A. Kundu, and B. P. Mandal Czech. J. Phys 54, 5 (2004).

[9] B. Basu-Mallick and B.P. Mandal, Phys. Lett. A 284, 231 (2001); B. Basu-Mallick, T. Bhattacharyya and B. P. Mandal, Mod. Phys. Lett. A 20 , 543 (2004).

[10] A. Khare and B. P. Mandal, Phys.Lett. A272, 53 (2000).

[11] B. P. Mandal, S. Gupta, Mod.Phys.Lett. A 25, 1723 (2010).

[12] B. P. Mandal, Mod. Phys. Lett. A 20, 655(2005).

[13] A. Ghatak and B. P. Mandal, J. Phys. A: Math. Theor. 45, 355301 (2012).

[14] B. P. Mandal and A. Ghatak, J. Phys. A: Math. Theor. 45, 444022 (2012).

[15] B. P. Mandal, B. K. Mourya, and R. K. Yadav (BHU), Phys. Lett. A 377, 1043 (2013).

[16] S. Longhi, J. Phys. A: Math. Theor. 44, 485302 (2011).

[17] C. M. Bender, D. W. Hook, P. N. Meisinger, Q. Wang, Phys.Rev.Lett. 104, 061601 (2010); C. M. Bender, D. W. Hook, K. S. Kooner J.Phys.A 43, 165201 (2010).

[18] A. Sinha, D. Dutta, P. Roy, Phys. Lett. A 375, 452 (2011).

[19] C. M. Bender, D. C. Brody, H. F. Jones, Phys.Rev. D 70, 025001 (2004).

[20] Z. H. Musslimani, K. G. Makris, R. El-Ganainy, and D. N. Christodoulides Phys.Rev. Lett. 100, 030402 (2008).

[21] C. E. Ruter, K. G. Makris, R. El-Ganainy, D. N. Christodoulides, M. Segev, D. Kip, Nature Physics 6 192, (2010);

[22] R. El-Ganainy, K. G. Makris, D. N. Christodoulides and Z. H. Musslimani Optics Letters 32, 2632 (2007).

[23] T. Kato, Perturbation Theroy of Linear Operators, Springer, Berlin, (1966).

[24] M.V. Berry, Czech. J. Phys. 54, 1039 (2004). 
[25] W. D. Heiss, Phys. Rep. 242, 443 (1994).

[26] A. Mostafazadeh Phys. Rev. Lett. 102, 220402 (2009).

[27] Z. Ahmed J. Phys. A: Math. Theor. 42, 472005(2009).

[28] A. Ghatak, J. A. Nathan, B. P. Mandal, and Z. Ahmed, J. Phys. A: Math. Theor. 45, 465305 (2012).

[29] Z. Ahmed J. Phys. A: Math. Theor. 45, 032004 (2012), Phys.Lett. A 324, 152 (2004), Phys. Lett. A 377, 957 (2013).

[30] A. Mostafazadeh, Phys. Rev. A 87, 012103 (2013).

[31] Zafar Ahmed, Carl M. Bender, M. V. Berry, J.Phys.A 38, L627 (2005).

[32] H. Ramezani, T. Kottos, R. El-Ganainy, D. N. Christodoulides, Phys. Rev. A 82, 043803 (2010).

[33] Y. D. Chong, Li Ge, and A. D. Stone, Phys. Rev. Lett. 106, 093902 (2011).

[34] G. Levai, P. Siegl, M. Znojil, J. Phys. A: Math. Theor. 42, 295201 (2009).

[35] K. Abhinav, A. Jayannavar, P. K. Panigrahi Ann. Phys. 331, 110 (2013).

[36] P. M. Morse and H. Feshback, Methods of Theoritical Physica, McGraw- Hill Book Company, (1953).

[37] S. Flugge, Practical Quantum Mechanics, Springer-Verlag Berlin Heidelberg, (1971). 